\title{
Proton aurora in the cusp during southward IMF
}

\author{
H. U. Frey and S. B. Mende \\ Space Sciences Laboratory, University of California, Berkeley, California, USA
}

S. A. Fuselier

Lockheed Martin ATC, Palo Alto, California, USA

T. J. Immel and N. Østgaard

Space Sciences Laboratory, University of California, Berkeley, California, USA

Received 24 January 2003; revised 19 March 2003; accepted 29 April 2003; published 8 July 2003.

[1] One of the most distinct aurorae in the high-latitude dayside region occurs at the footprint of the cusp. The Spectrographic Imager SI-12 channel on the IMAGE spacecraft allows for long-duration observations of the proton aurora in this region and thus enables morphological and quantitative studies of the behavior of the proton aurora under different external solar wind conditions. Here we extend an earlier study of the proton aurora in the cusp during northward interplanetary magnetic field (IMF) conditions [Frey et al., 2002] to southward IMF conditions. We find a very similar dependence of the cusp aurora's location in magnetic local time (MLT) on the IMF $\mathrm{B}_{y}$ component, with locations at prenoon for negative $\mathrm{B}_{y}$ and postnoon for positive $\mathrm{B}_{y}$. We also confirm the previously established dependence of the proton aurora brightness on the solar wind dynamic pressure. However, for similar solar wind pressures the proton aurora is brighter for the southward IMF case than for the northward IMF case. The other major difference between southward and northward IMF conditions is the very strong dependence of the latitude location on the magnitude of the IMF $\mathrm{B}_{z}$ for southward IMF. We discuss both differences in terms of the location of the reconnection site at the magnetopause that is connected to the ionosphere along cusp magnetic field lines. INDEX TERMS: 2704 Magnetospheric Physics: Auroral phenomena (2407); 2724 Magnetospheric Physics: Magnetopause, cusp, and boundary layers; 2784 Magnetospheric Physics: Solar wind/magnetosphere interactions; 7835 Space Plasma Physics: Magnetic reconnection; 2455 Ionosphere: Particle precipitation; KEYWORDS: proton aurora, interplanetary magnetic field (IMF), cusp, reconnection, solar wind

Citation: Frey, H. U., S. B. Mende, S. A. Fuselier, T. J. Immel, and N. Østgaard, Proton aurora in the cusp during southward IMF, J. Geophys. Res., 108(A7), 1277, doi:10.1029/2003JA009861, 2003.

\section{Introduction}

[2] The objective of the IMAGE mission is to improve the understanding of the interaction between the solar wind, magnetosphere, and ionosphere. One signature of this interaction is the occurrence of aurorae which are observed by the Far Ultraviolet Instrument (FUV) on IMAGE [Mende et al., 2000]. The major advantage of this instrument compared with previously flown ultraviolet imagers is the capability of the Spectrographic Imager SI-12 channel to provide the first global observations of the proton aurora by observing the Doppler-shifted component of Lyman- $\alpha$ radiation from proton precipitation. Precipitating proton contributions to the overall energy deposition into the ionosphere can at certain locations (cusp, dusk region) and times (before substorm onset around midnight) account for a significant portion $(20-30 \%)$ of the overall energy input [Frey et al., 2001; Hubert et al., 2002].

Copyright 2003 by the American Geophysical Union. 0148-0227/03/2003JA009861\$09.00
[3] A very distinct aurora at the high-latitude, dayside region occurs at the footprint of the cusp [Smith and Lockwood, 1996]. In situ electron and ion measurements have shown that the location and spectrum of particle precipitation in the cusp depend on the IMF orientation [Newell et al., 1989] and that they are an indicator of reconnection at the magnetopause [see, e.g., Onsager et al., 1993]. The particle precipitation into the cusp shows differences depending on the external solar wind magnetic field orientation. A velocity (energy)-latitude dispersion can be found, with higher-energy particles precipitating closer to the reconnection site, which is the higher-energy particles at higher latitudes for northward IMF and at lower latitudes for southward IMF [Onsager et al., 1995]. This energy-latitude dispersion is a signature of the underlying reconnection process at high/low latitudes and the transport of reconnected field lines with the less energetic particles to lower/ higher latitudes [Woch and Lundin, 1992; Onsager and Lockwood, 1997].

[4] Precipitating particles create aurora and such auroral forms at the cusp footprint were studied using data from 
ground-based meridian scanning photometer and all-sky camera observations [Sandholt et al., 1998]. The rotation of the Earth and the relative motion of the site to different local times allowed for the study of the local time dependence on the IMF east-west $\mathrm{B}_{y}$ component [Sandholt and Farrugia, 1999]. The north-south extent of the observations allowed for the investigation of latitudinal motions with respect to the IMF $\mathrm{B}_{z}$ component [Sandholt et al., 1994]. Several studies demonstrated that the cusp footprint occurred at lower latitudes for southward IMF $\mathrm{B}_{z}$ than for positive $\mathrm{B}_{z}$ and described associated particle precipitation (latitude dispersion) and convection (shift of convection boundaries) characteristics [McCrea et al., 2000; Sandholt et al., 2001]. The combination of ground-based radar and space-based ultraviolet observations confirmed the equatorward motion of the auroral oval and the excitation of ionospheric convection after a southward turn of the IMF [McWilliams et al., 2001]. HF radar observations showed an equatorward migration of the HF radar cusp during southward IMF but no change during northward IMF [Yeoman et al., 2002].

[5] These investigations were recently extended with results from the FUV instrument on IMAGE, which is capable of global observations of the auroral zone and of distinguishing between proton and electron produced aurora. In a statistical study, Frey et al. [2002] demonstrated the dependence of the proton aurora brightness at the cusp footprint on the solar wind dynamic pressure. That study also showed the dependence of the cusp magnetic local time (MLT) location on IMF $\mathrm{B}_{y}$, consistent with earlier observations [Moen et al., 1999] and with theoretical considerations of the partial penetration of the IMF $\mathrm{B}_{y}$ into the magnetosphere [Cowley, 1981]. Furthermore, the study demonstrated that the proton precipitation can account for about $30 \%$ of the total energy input into the cusp. This indicated that optical aurora observations which were attributed largely to electron precipitation should be corrected for the contribution of proton excitation to the electron aurora.

[6] A few case studies were used in another report to investigate the morphological changes of the cusp aurora as a function of the orientation of the interplanetary $\mathrm{B}_{z}$ component [Fuselier et al., 2002]. It was shown that the spot-like cusp signature during northward IMF changes morphology, becomes elongated in MLT, and merges with the dayside auroral oval with the southward turning of the IMF. These results were discussed in the context of antiparallel reconnection (at the high-latitude lobe magnetopause during northward IMF) and possible component reconnection (at the low-latitude magnetopause during southward IMF).

[7] In another single event study the response of the Doppler-shifted Lyman- $\alpha$ emission in the cusp to a shortlived southward turning of the IMF during a period of strongly enhanced solar wind plasma density was studied [Lockwood et al., 2003]. The intensity of the cusp emission varied systematically with the IMF clock angle and theoretical predictions of the intensity of Lyman- $\alpha$ emission were confirmed by the space-based observations.

[8] In this study we will investigate the location and intensity of the cusp proton aurora under southward IMF conditions and compare the results with the previous northward IMF study [Frey et al., 2002]. After a brief summary of instrumentation and analysis techniques, we discuss a few individual observations and describe the results of a statistical study of 15 cases and their correlation with the corresponding solar wind conditions.

\section{Instrumentation and Data Analysis}

[9] The IMAGE satellite is in an elliptical orbit of $1000 \times$ $45000 \mathrm{~km}$ altitude. The Far Ultra-Violet imager (FUV) consists of three imaging instruments and observes the aurora for 5-10 s during every $2 \mathrm{~min}$ spin period [Mende et al., 2000]. Major properties such as fields of view, spatial resolution, and spectral sensitivity were validated by inflight calibrations with stars [Frey et al., 2003]. The Wideband Imaging Camera (WIC) has a passband of $140-180 \mathrm{~nm}$. It measures emissions from the $\mathrm{N}_{2} \mathrm{LBH}-$ band and atomic NI lines, with small contributions from the OI $135.6 \mathrm{~nm}$ line. The proton aurora imaging Spectrographic Imager channel (SI-12) is sensitive to the Doppler-shifted Lyman- $\alpha$ emission around $121.8 \mathrm{~nm}$ from charge-exchanging precipitating protons. It is mostly sensitive to proton precipitation in the energy range of $2-8 \mathrm{keV}$, with very low sensitivity below $1 \mathrm{keV}$ [Gérard et al., 2000, 2001]. The oxygen imaging Spectrographic Imager channel (SI-13) has a passband of $5 \mathrm{~nm}$ around the $135.6 \mathrm{~nm}$ doublet of oxygen OI emission. The measured signal is a combination of OI and some contribution from lines in the $\mathrm{N}_{2} \mathrm{LBH}$ emission band ( $20-50 \%$ depending on electron energy).

[10] Solar wind parameters for this study were obtained through CDAWeb from the WIND, ACE, and Geotail spacecraft. Over this study period (summer/fall 2000) the Geotail apogee of 30 Earth radii was located between 1200 and 1600 magnetic local time. At the start of the study interval WIND was located at $40 \mathrm{R}_{e}$ and 0900 MLT, and at the end of the interval WIND was located at $250 \mathrm{R}_{e}$ and 0600 MLT, having made two transits through the magnetosphere. Measurements within the magnetosphere and whenever WIND was more than $65 \mathrm{R}_{e}$ away from the Sun-Earth line were discarded for this study. In those cases, ACE data were used. All solar wind properties were propagated to their most likely ionospheric impact times using the method described in [Jacobsen et al., 1995]. All magnetic field data were transformed into the GSM coordinate system.

[11] In contrast to the earlier investigation [Frey et al., 2002], where a localized auroral emission poleward of the dayside aurora was investigated, a different data selection method was used in this study. During the time period of 23 June to 16 September 2000 (days of year 175-260), extended periods of at least $20 \mathrm{~min}$ of southward IMF and enhanced solar wind particle density $\left(>5 \mathrm{~cm}^{3}\right)$ were selected. One additional time period on 28 October 2000 was included because FAST measurements were available during a transit through the southward IMF cusp. The dayside region in the SI-12 images was searched for the appearance of the proton aurora at the cusp footprint. Fifteen clear cases and a total of 32 hours were found (Table 1). Figure 1 shows examples from 4 different days, when the extended feature of strong proton aurora could be observed within the dayside auroral oval. For comparison we also show the corresponding WIC images below the SI-12 images, which show a morphologically similar signature.

[12] Each individual time sequence of images was analyzed in a manner similar to that employed in the earlier 
Table 1. Summary of All Events Used for This Study ${ }^{\mathrm{a}}$

\begin{tabular}{|c|c|c|c|c|}
\hline Date and Time & Pressure & $\mathrm{B}_{x}$ & $\mathrm{~B}_{y}$ & $\mathrm{~B}_{z}$ \\
\hline $2000-175,1301-1358$ & $1.8-8.4(6.2)$ & 2 to $16(11)$ & -14 to $-0(-8)$ & -14 to $0(-8)$ \\
\hline $2000-176,0519-0612$ & $5.0-10.6(7.5)$ & 8 to $12(10)$ & -8 to $3(-6)$ & -6 to $1(-3)$ \\
\hline $2000-192,1823-1846$ & $7.4-11.4(9.7)$ & -11 to $10(-2)$ & 8 to 14 (11) & -9 to $0(-6)$ \\
\hline $2000-193,0951-1218$ & $5.0-17.8(10.6)$ & -3 to 7 (3) & 8 to $24(10)$ & -5 to $0(-3)$ \\
\hline $2000-208,1656-2025$ & $1.8-7.6(4.6)$ & -4 to $2(-1)$ & -4 to $6(2)$ & -9 to $0(-6)$ \\
\hline $2000-210,1155-1259$ & $6.9-9.9(8.6)$ & 0 to 8 (3) & 0 to 19 (14) & -18 to $0(-7)$ \\
\hline $2000-211,0129-0227$ & $2.4-7.0(4.9)$ & 6 to $10(8)$ & -10 to $-8(-9)$ & -6 to $1(-3)$ \\
\hline $2000-225,0704-1201$ & $2.0-6.1(3.7)$ & -6 to $12(2)$ & -10 to $29(17)$ & -30 to $-14(-24)$ \\
\hline $2000-236,1331-1458$ & $4.2-5.5(4.8)$ & 3 to 9 (7) & 3 to $6(4)$ & -6 to $-1(-4)$ \\
\hline $2000-237,0351-0624$ & $3.0-5.3(3.9)$ & -5 to $4(-1)$ & 6 to $10(9)$ & -7 to $-2(-4)$ \\
\hline $2000-241,0847-1129$ & $3.0-6.2(4.4)$ & -10 to $-2(-5)$ & -3 to 9 (4) & -7 to $0(-3)$ \\
\hline $2000-256,1708-1955$ & $2.0-3.2(2.5)$ & -3 to $4(0)$ & -8 to $6(-2)$ & -10 to $1(-6)$ \\
\hline $2000-259,1950-2156$ & $3.5-6.0(4.6)$ & -5 to $2(-1)$ & -8 to $10(1)$ & -14 to $1(-7)$ \\
\hline $2000-260,2100-2252$ & $2.0-6.0(3.2)$ & -9 to $2(-3)$ & -13 to $5(-3)$ & -17 to $0(-14)$ \\
\hline $2000-302,0933-1237$ & $2.1-12.8(8.3)$ & -7 to $1(-4)$ & 2 to $11(6)$ & -11 to $0(-5)$ \\
\hline
\end{tabular}

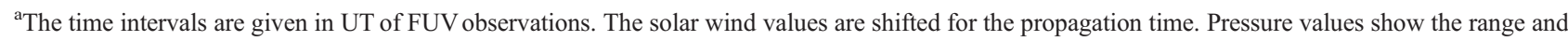
mean in $\mathrm{nPa}$. The magnetic field values show the range and mean values in $\mathrm{nT}$, rounded to the nearest integer.

investigation. The mean count rate in a region of $5 \times 5$ pixels (about $500 \times 500 \mathrm{~km}$ from apogee) around the brightest pixel was determined, as well as its location in MLT and geomagnetic latitude. The WIC and SI-13 instrument responses were then determined for the same location. The average of the preexisting (in case of IMF orientation change) or surrounding (during steady southward IMF) dayside auroral oval was subtracted from the instrument signal. Whenever the IMF turned northward the analysis was stopped.

\section{FUV Observations}

\subsection{June 2000}

[13] WIND observations on 24 June $2000($ doy $=176)$ show a period of $50 \mathrm{~min}$ of southward IMF (Figure 2). Around
0520 UT the IMF $B_{z}$ (solid line) changed from $+10 \mathrm{nT}$ to values around $-5 \mathrm{nT}$ and then slowly increased towards positive values again. The solar wind pressure during that time was originally around $9 \mathrm{nPa}$ until it dropped below 6 $\mathrm{nPa}$ at 0554 . The mean count rate in the brightest dayside region of SI-12 images increased dramatically with the change to negative $\mathrm{B}_{z}$ and then slowly decreased until the end of the time period. The geomagnetic latitude of the bright proton aurora slowly changed from $75^{\circ}$ to less then $70^{\circ}$. The MLT location did not change considerably during that time period.

\subsection{July 2001}

[14] FUV observations on 10 July 2000 (doy $=192)$ show the very obvious morphological change of the dayside aurora with changes of the IMF $B_{z}$ (Figure 3). Geotail

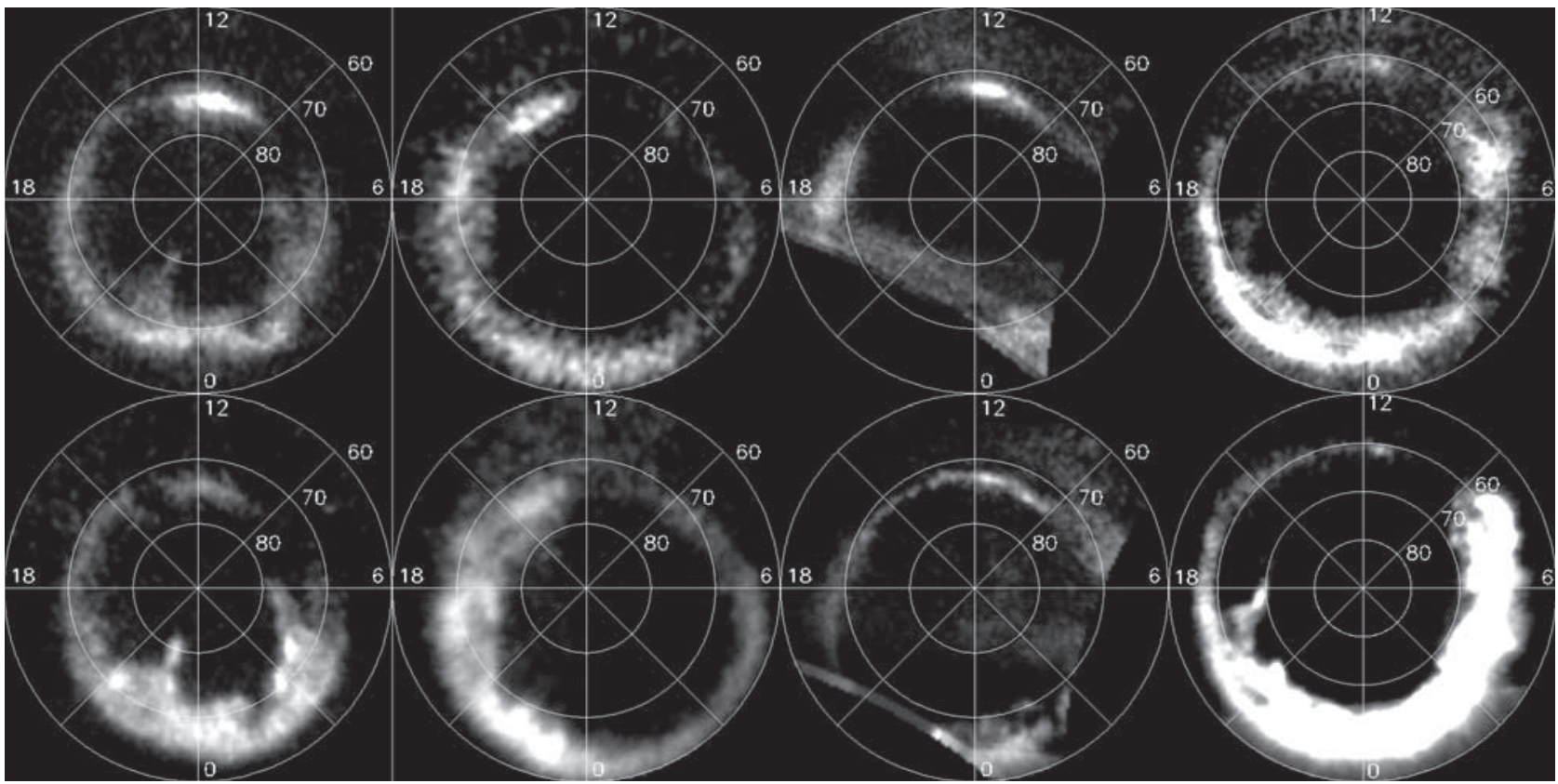

Figure 1. Examples of observations of SI-12 (top row) and the corresponding WIC images (bottom row) for 4 different days in 2000. The original images were remapped into a MLT-latitude grid with local noon at the top, midnight at the bottom, and dawn to the right of each image. The lowest latitude in the right images is $50^{\circ}$, whereas it is $60^{\circ}$ in all other images. 

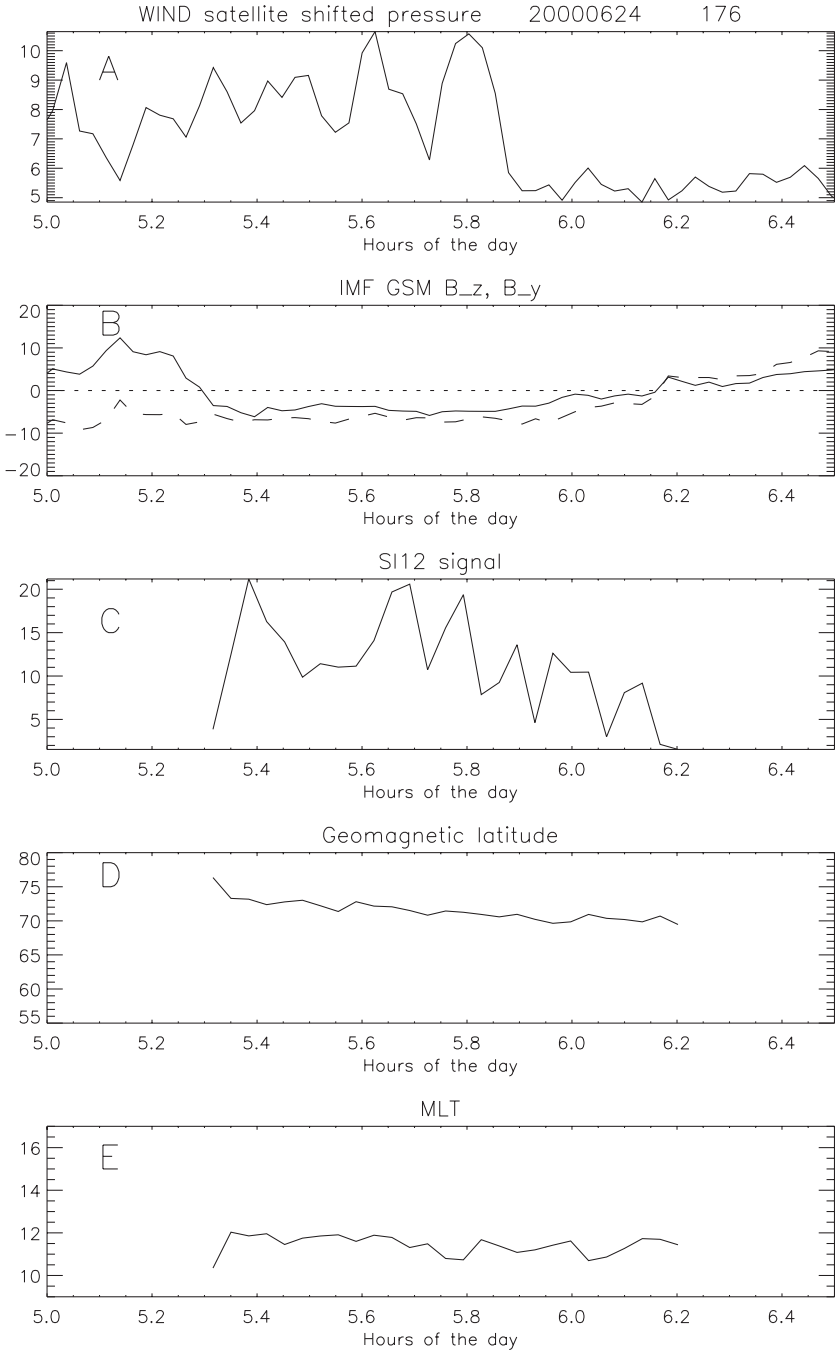

Figure 2. Summary of ultraviolet observations and related, propagated solar wind parameters for 24 June 2000 (doy = 176). The panels from top to bottom show the time shifted solar wind dynamic pressure (a), IMF-GSM $\mathrm{B}_{z}$ (solid line) and $\mathrm{B}_{y}$ (dashed line) components (b) (WIND measurements), the average proton aurora emission (c) (instrument counts), geomagnetic latitude (d) and MLT (e) of the brightest pixel in the SI-12 images.

measurements in the solar wind were used because WIND was in the magnetosphere. The IMF $\mathrm{B}_{z}$ was positive before 1823 and the typical separated, spot-like proton aurora was observed poleward of the auroral oval at about 1500 MLT and $83^{\circ}$ latitude. $\mathrm{B}_{z}$ changed suddenly to $-5 \mathrm{nT}$ and the spot-like feature disappeared, and the brightness of the originally dim dayside auroral oval intensifies, centered at 1400 MLT and $76^{\circ}$ latitude. $B_{z}$ stayed negative for about 22 min until as suddenly as before it changed to $+4 \mathrm{nT}$. $\mathrm{B}_{x}$ and $\mathrm{B}_{y}$ stayed relatively constant at $-1 \mathrm{nT}$ and $11 \mathrm{nT}$, respectively. Following the northward turn of $\mathrm{B}_{z}$ the spotlike feature appeared at almost the same location as before. These observations confirm previous results of longitudinally wider but latitudinally narrower cusp during southward IMF compared to the northward IMF case [Newell et al., 1989; Fuselier et al., 2002].
[15] Figure 3 demonstrates the equatorward motion of the bright proton aurora during the southward IMF period 1823 1846. Over the course of $20 \mathrm{~min}$ the aurora migrates equatorward at an average rate of $0.027^{\circ} / \mathrm{min} / \mathrm{nT}$, a value very similar to the $0.02^{\circ} / \mathrm{min} / \mathrm{nT}$ given by Yeoman et al. [2002].

\subsection{August 2000}

[16] The observations on 12 August $2000($ doy $=225)$ represent an example of extreme southward IMF conditions with a minimum as low as $-30 \mathrm{nT}$ (ACE data, Figure 4). An example of FUV images is given in the right panels of Figure 1. The IMF $B_{z}$ has been negative for 1.5 hours prior to the FUV turn-on after IMAGE left the radiation belt at 0702. In contrast to the extreme magnetic field conditions the solar wind pressure was rather low with values around $5 \mathrm{nPa}$. This is still higher than the normal solar wind pressure of $1.5 \mathrm{nPa}$ but required to produce a reasonable signal in the SI-12 imager [Frey et al., 2002]. This pressure even decreased to $3 \mathrm{nPa}$ over the next hours. The decreasing solar wind dynamic pressure is accompanied by a similar decrease of the SI-12 signal, again confirming the relationship between the proton aurora signal and the external solar wind pressure.

[17] The most remarkable feature of that time period is the location of the bright proton aurora in magnetic latitude. The extended period of strong southward IMF had pushed the cusp footprint down below $60^{\circ}$ latitude and the increase of $\mathrm{B}_{z}$ from -30 to $-13 \mathrm{nT}$ was accompanied by a slow poleward motion ( 3 degrees per hour) of the cusp aurora to locations around $65^{\circ}$ latitude. This poleward motion contradicts the results from HF radar observations which showed an equatorward motion during southward IMF [Yeoman et al., 2002].

\subsection{July 2000}

[18] The observations on 26 July $2000($ doy $=208)$ are summarized in Figure 5. Even before the FUV turn-on the IMF $\mathrm{B}_{z}$ component had been negative for 5 hours. The solar wind pressure was $2 \mathrm{nPa}$ at $1700 \mathrm{UT}$, increased to $3 \mathrm{nPa}$ after 1800 UT, and suddenly increased to $7 \mathrm{nPa}$ at 1900 UT. The pressure increases were accompanied by increases in the SI12 signal, but after the initial increase the proton aurora signal decreased again. The IMF $\mathrm{B}_{z}$ was rather stable between -5 and $-9 \mathrm{nT}$ over the 3.5 hours interval and in this particular case there was a slow increase of the bright proton aurora latitude with small jumps at the pressure increases. The proton aurora MLT location slowly changed to earlier MLT though we would have rather expected a small change towards later hours with the change of $\mathrm{B}_{y}$ to higher positive values.

\subsection{October 2000}

[19] The time interval of 28 October $2000($ doy $=302)$ 0933-1237 was added to this study because it coincided with a FAST pass through the cusp at 12.5 MLT (Figure 6), which is summarized in Figure 7. The particle observations show a very clear example of energy-latitude dispersion of precipitating protons during southward IMF conditions [Smith and Lockwood, 1996]. FAST crosses the cusp from lower latitudes, encountering the higher energy protons of about $2 \mathrm{keV}$ mean energy first. As the spacecraft moves poleward it observes progressively lower proton energies. Further poleward it measured ions of just a few $100 \mathrm{eV}$ in the plasma mantle region. Similar examples of particle distributions and identifications of precipitation regions 

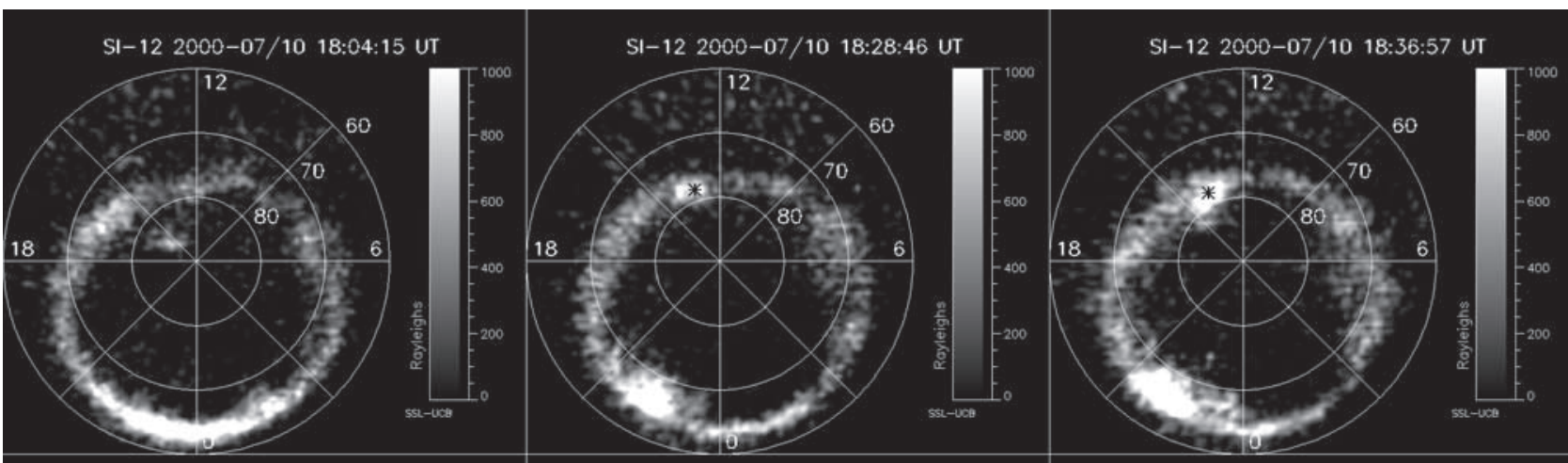

SI-12 2000-07/10 18:43:04 UT

SI-12 2000-07/10 18:45:07 UT
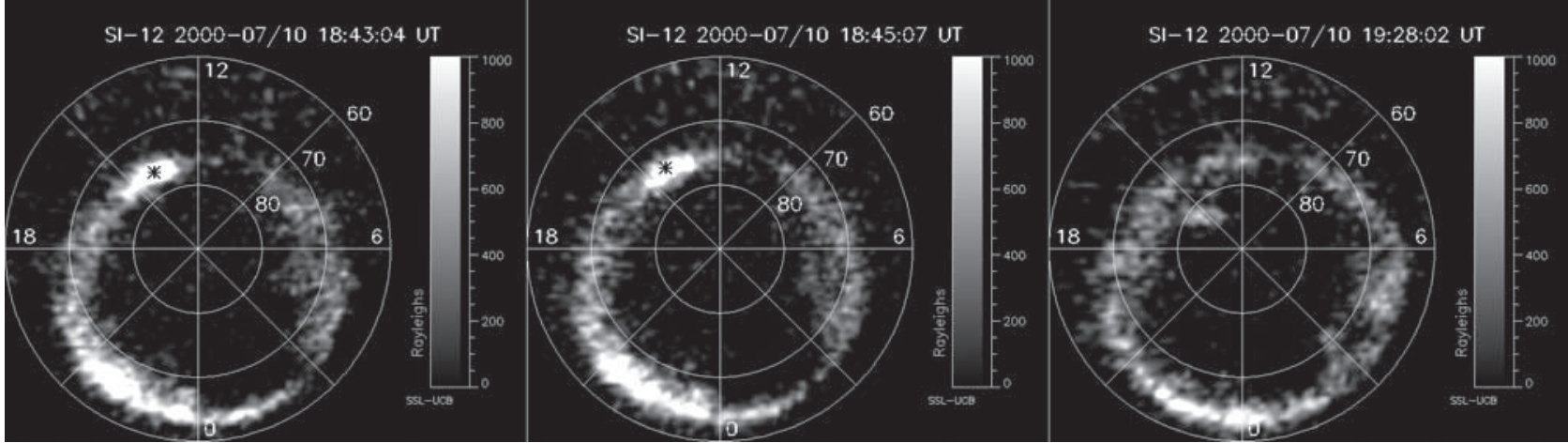

Figure 3. Examples of observations on 10 July 2001. The solar wind $\mathrm{B}_{z}$ component was originally northward (1804), changed to southward (1823-1846) and back to northward again (1928), causing the obvious morphological changes in the cusp proton aurora. During the southward IMF interval the location of the brightest pixel of proton aurora is given with the black asterisk.

are given by Newell et al. [1991]. The energy-latitude dispersion is the result of low-latitude reconnection and the subsequent transport of reconnected field lines to higher latitudes [Onsager and Lockwood, 1997]. This transport causes higher-energy protons to arrive first at lower latitudes and lower-energy protons to arrive later in the ionosphere at higher latitudes [Smith and Lockwood, 1996; Onsager et al., 1993].

[20] The magnetic field disturbance in the cross-track direction (green line, top panel) shows two major fieldaligned current regions. The more poleward region of measurements between 1105:40 and 1106:10 (positive slope) shows a downward current region which coincides with the very low ion and electron energy $(<500 \mathrm{eV}$ and $<100 \mathrm{eV}$, respectively) mantle precipitation. Equatorward of this, there is an upward current region (1105:20-1105:40, negative slope) with the higher energy ion precipitation, and the signature of latitude-energy dispersed ion precipitation in the cusp. The SI-12 count rates along the FAST footprint (bottom panel of Figure 7) show the highest proton aurora signal in the upward current region with the higher energy proton precipitation. The more poleward region of mantle precipitation has lower proton energies and energy fluxes and results in lower SI-12 signal.

\section{Statistical Summary}

\subsection{Location of Emission}

[21] All examples of proton aurora (section 2) occur between $58^{\circ}$ and $78^{\circ}$ geomagnetic latitude (Figure 8). Their locations were distributed between 9.8 and 15.9 hours MLT. The data points from 12 August, the extreme southward
IMF case, will be plotted in gray to distinguish them from the bulk of other observations.

[22] The spatial distribution of the proton aurora during southward IMF conditions is very similar to that determined from the northward IMF cases [Frey et al., 2002], but there is a significant latitudinal offset. The mean latitude location of $70.1^{\circ}$ is $9^{\circ}$ lower compared with the mean northwardIMF cusp latitude of $79.2^{\circ}$. An outstanding feature of this distribution is the group of locations around $60^{\circ}$ latitude and 12.5 MLT. All these points are from 12 August 2002 when the very strong southward IMF pushed the bright proton aurora at the cusp footprint dramatically equatorward. The mean local time of 12.9 hours for the southward IMF cusp footprint is one hour later compared with the 11.7 hours location of the northward IMF cusp footprint. This is a special feature of the data set and is not caused by the southward IMF. More discussion on this will be given in section 5 .

\subsection{Dependence of Emission on Solar Wind Magnetic Field}

[23] The propagated solar wind measurements were used to correlate the location and intensity of the localized emissions with the solar wind magnetic field and dynamic pressure. The dependence of the geomagnetic latitude and the FUV emission brightness of the bright proton aurora on the IMF $B_{z}$ values are given in Figure 9. There is a clear dependence of the latitude location on $\mathrm{B}_{z}$ with a correlation coefficient of 0.81 and a least-square fitted dependence of

$$
\text { latitude }=73.8^{\circ}+0.45 B_{z},
$$

with $\mathrm{B}_{z}$ here given in $\mathrm{nT}$. The standard deviation of the linear parameter is 0.01 . This result is similar to the 

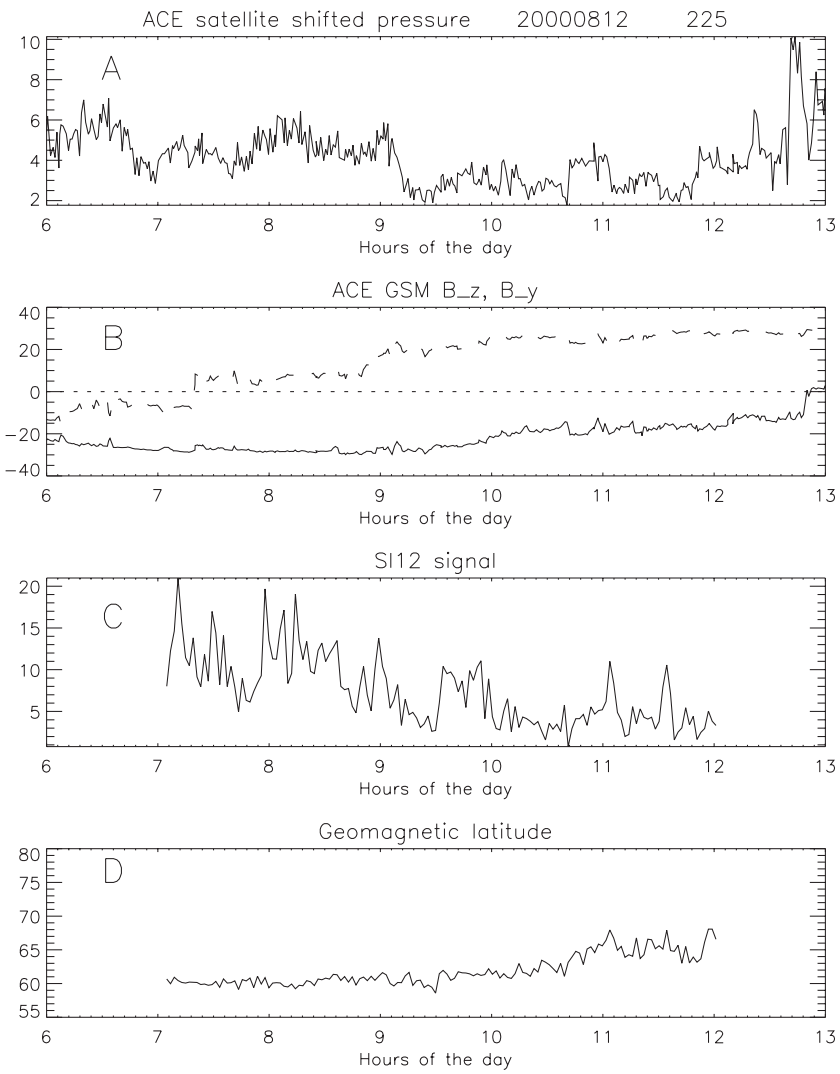

$M L T$

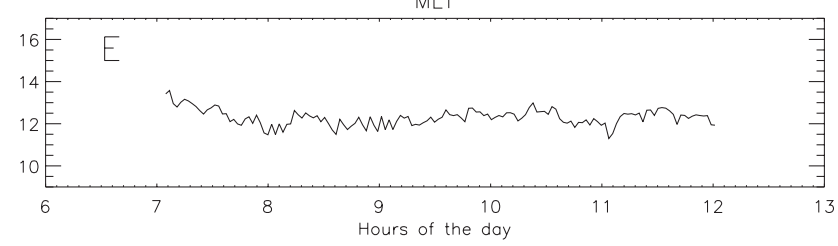

Figure 4. Summary of ultraviolet observations and related solar wind parameters (ACE measurements) for 12 August 2000. The format is similar to Figure 2.

observation of an 0.7 correlation between the cusp latitude and $\mathrm{B}_{z}$ [Newell et al., 1989]. However, our relationship (solid line) is different from the $77.0^{\circ}+0.76 \mathrm{~B}_{z}$ (dashed line) found in their study, though both graphs do not show huge differences (Figure 9). Newell et al. [1989] focused on the equatorward boundary of the cusp and found it at higher latitudes than we found the proton aurora, but it is important to note that in their study IMF $B_{z}$ was not less than $-10 \mathrm{nT}$. Even if we exclude the data from 12 August we find the same linear trend offset in latitude. One difference is our larger range of $\mathrm{B}_{z}>-30 \mathrm{nT}\left(\mathrm{B}_{z}>-18 \mathrm{nT}\right.$ without 12 August) compared with their cases of $\mathrm{B}_{z}>-5 \mathrm{nT}$. On the other hand their Figure 4 shows cusp locations at 1200 MLT between $71^{\circ}$ and $74^{\circ}$ which match our observations. However, a different study found equatorward boundary locations for southward IMF of $75.5^{\circ}+0.61 \mathrm{~B}_{z}$ which is much closer to our result [Carbary and Meng, 1986]. This disagreement may demonstrate the importance of the particular data set even in a statistical study.

[24] A study of ground-based optical and magnetic observations found a dependence of $75.0^{\circ}+0.5 \mathrm{~B}_{z}$ between the latitude of cusp aurora and $\mathrm{B}_{z}$ which is much closer to our result [Sandholt et al., 1994]. This agreement between the optical data may indicate that there is a difference in boundary and centroid determination between particle observations and optical observations. In general the equatorward boundary of the proton aurora could be a better property than the aurora centroid and better related to the IMF, if equatorward motion is mixed with a size increase of the cusp aurora. However, the centroid was used in the previous study and we want to use a similar approach for comparison of the results.

[25] There is no indication that the intensity of the FUV emission is controlled by the magnitude of $\mathrm{B}_{z}$ (correlation coefficient $R=0.06$ ). There appears to be a bias of lower brightness for stronger southward IMF, but this bias is introduced by the data from 12 August with extreme magnetic fields but only small solar wind pressure.

[26] Figure 10 summarizes the dependence of the magnetic local time location and SI-12 signal on the value of IMF $\mathrm{B}_{y}$. In this particular data set we find only a small correlation coefficient between the $\mathrm{B}_{y}$ and MLT-location of 0.28 (gray line). If the data from 12 August 2002, which were measured during the extreme $\mathrm{B}_{y}$ and $\mathrm{B}_{z} \mathrm{IMF}$ con-
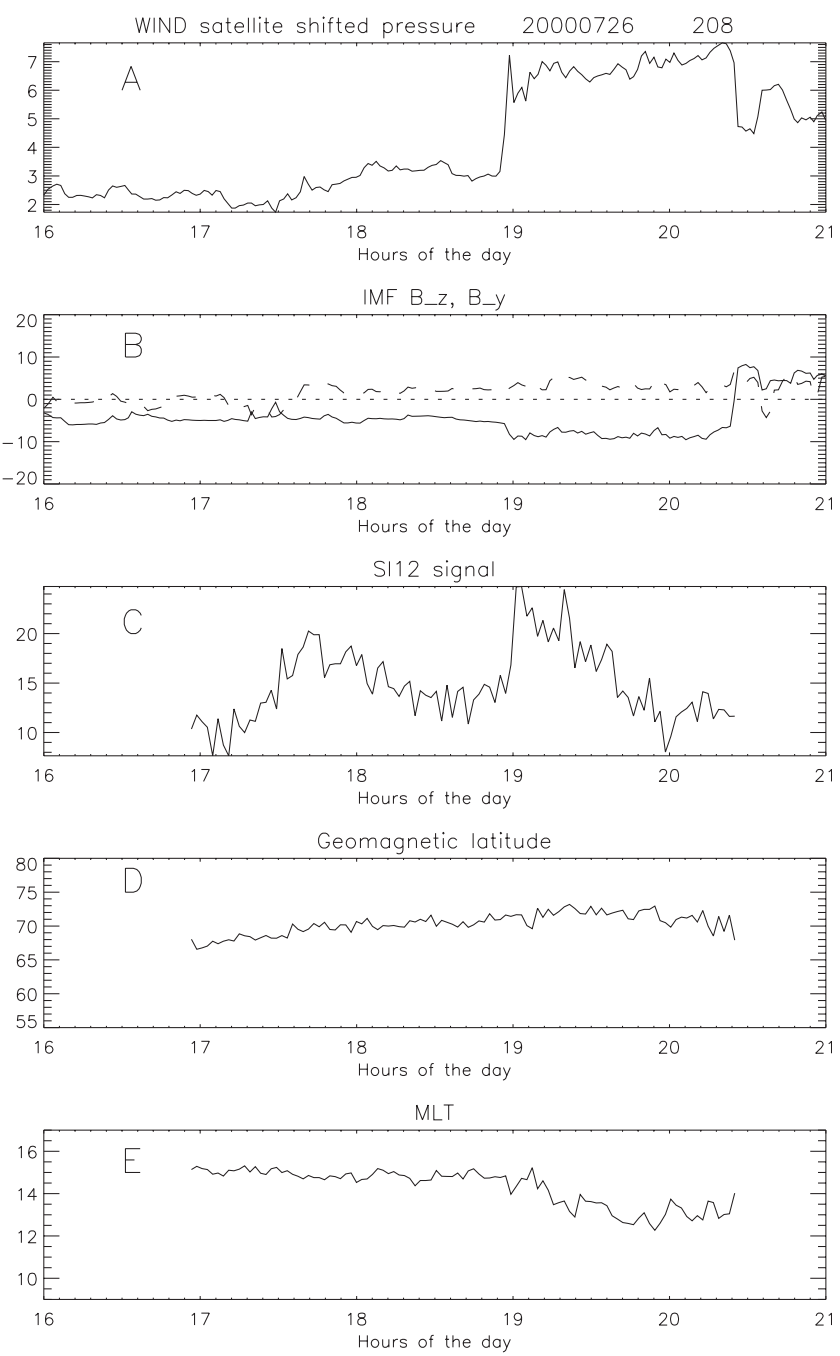

Figure 5. Summary of solar wind and FUV observations on 26 July 2000 (WIND measurements). The format is similar to Figure 2. 


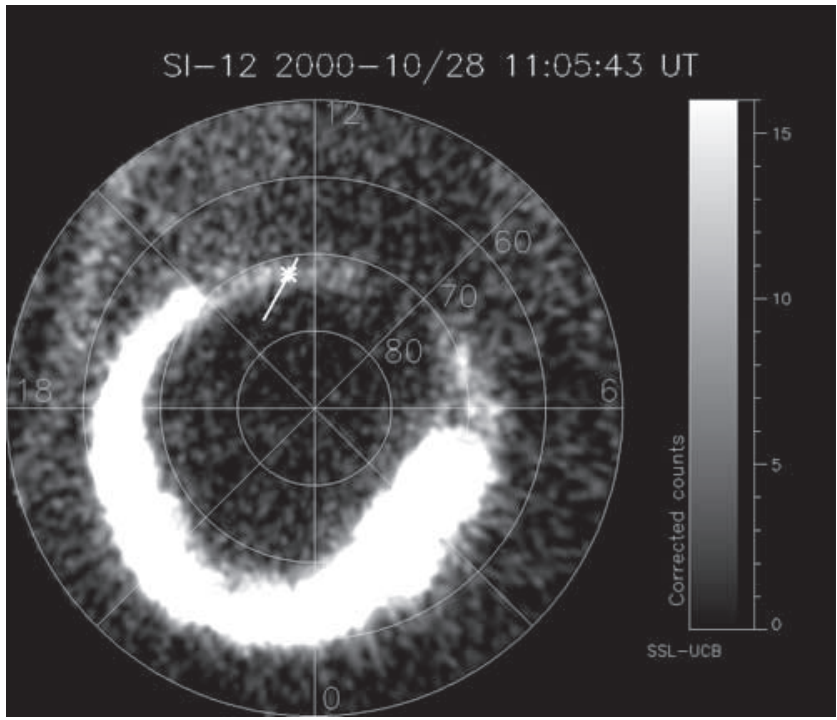

Figure 6. Mapped SI-12 image taken on 28 October 2000 at 1105:43 with the footprint along the FAST satellite track from $1 \mathrm{~min}$ before to $3 \mathrm{~min}$ after image integration. At image integration, FAST is just at the poleward edge of the cusp proton aurora and this footprint location is given by the small asterisk.

ditions, are excluded (black line), we obtain a much better correlation of 0.60 and a least square fitted relation of

$$
M L T=12.6 \text { hours }+0.120 B_{y}
$$

with $\mathrm{B}_{y}$ given in $\mathrm{nT}$. It is interesting to note that the linear coefficient of 0.120 agrees well with the one for the northward IMF cusp of 0.127 [Frey et al., 2002]. There is no apparent relationship between the $\mathrm{B}_{y}$ component and the brightness of the proton aurora.

[27] The range of $B_{x}$ values was -11 to $+16 \mathrm{nT}$. There is no indication of any correlation between the magnitude of IMF $B_{x}$ and any other of our investigated cusp aurora properties, including the latitude position similar to the results given by Newell et al. [1989]. The largest absolute value of all correlation coefficients was -0.32 between the MLT location and $\mathrm{B}_{x}$. It is most likely that this weak dependence is rather created by the MLT-B $_{y}$ dependence and the general IMF Parker spiral geometry instead of a true $\mathrm{B}_{x}$ dependence. Such a bias would indeed create an anticorrelation with $\mathrm{B}_{x}$ if there is a positive correlation with $\mathrm{B}_{y}$. We therefore do not consider any significant correlation between MLT location and $\mathrm{B}_{x}$.

\subsection{Dependence of Emission on Solar Wind Dynamic Pressure}

[28] Figure 11 summarizes the dependence of the SI-12 signal on the solar wind dynamic pressure. Observations were made during periods of up to $18 \mathrm{nPa}$ pressure. As in the case of northward IMF proton aurora observations in the cusp [Frey et al., 2002] there is a reasonable correlation between both quantities of 0.55 and a least-square fitted dependence of

$$
S I 12=2.0+1.51 p_{d y n}
$$

with pressure given in $\mathrm{nPa}$ and signal given in SI-12 counts. Here it has to be emphasized that several examples in section 3 showed the trend of decreasing proton aurora brightness during steady solar wind pressure. Only shortly after pressure increases is the brightness really determined by the instantaneous pressure value, while later there is behavior depending on the previous history. Such a behavior inhibits a better correlation than the one given above. We tested this hypothesis by using only those data points within 2 min after a pressure increase of more than $1 \mathrm{nPa}$ (57 data points). In that case we get a better correlation of 0.68 and the linear term in equation (3) increases to 1.87 .

\section{Discussion}

[29] Since the beginning of permanent observations in early June 2000, the SI-12 instrument frequently observed bright proton aurora in the dayside/cusp region. We limited the present study to observations in approximately the same time interval as in the previous study (late summer, fall) [Frey et al., 2002]. As the cusp footprints move slightly with the dipole tilt and season [Coleman et al., 2000], we did not want to mix very different dipole tilt and solar illumination conditions into our statistical analysis. For instance, the reduced ionospheric conductivity around winter solstice could have an influence on the intensity of the proton precipitation and that should be the topic of a different study.

[30] The mapping code which was developed for earlier studies [Fuselier et al., 2002; Frey et al., 2002] was used for several of our present cases and confirmed that the bright proton aurora cusp region maps to the low-latitude dayside magnetopause. We do not show specific examples in this paper as several have been shown in the previously mentioned papers. This result confirms that the cusp during southward IMF is magnetically connected to the low-latitude region and is created by reconnection of dayside magnetic field lines with the magnetosheath field [Fuselier et al., 2002].

[31] The present study confirms two major results of the earlier study of cusp proton aurora during northward IMF conditions. The magnetic local time location of the bright proton aurora at the cusp footprint is determined by the IMF $\mathrm{B}_{y}$ component with an average change of absolute location of 0.120 hours/nT (equation (2)). As was mentioned earlier, the mean location of the proton aurora in this study is 1 hour later than for the northward IMF case. However, as the mean of $\mathrm{B}_{y}$ here is $+4.7 \mathrm{nT}$ compared with the mean value of $-0.9 \mathrm{nT}$ in the previous study this result further confirms the trend of the MLT motion with $\mathrm{B}_{y}$ [see, e.g., Sandholt and Farrugia, 1999].

[32] The second result is the dependence of the proton aurora brightness on the solar wind dynamic pressure. In both cases a reasonably good correlation of 0.65 (previous study) and 0.55 (this study) was found. As mentioned in several cases, the correlation between the proton aurora brightness and the solar wind dynamic pressure decreased with time, since it was found that for constant solar wind dynamic pressure there was a slow decrease of the proton aurora brightness (see, e.g., Figure 5). Such a trend counteracts the general result of an increased brightness whenever the external pressure increased. However, this result in itself is important as it shows that for constant external conditions the brightness of the proton aurora at the cusp footprint is 


\section{FAST Particles Orbit 16608}
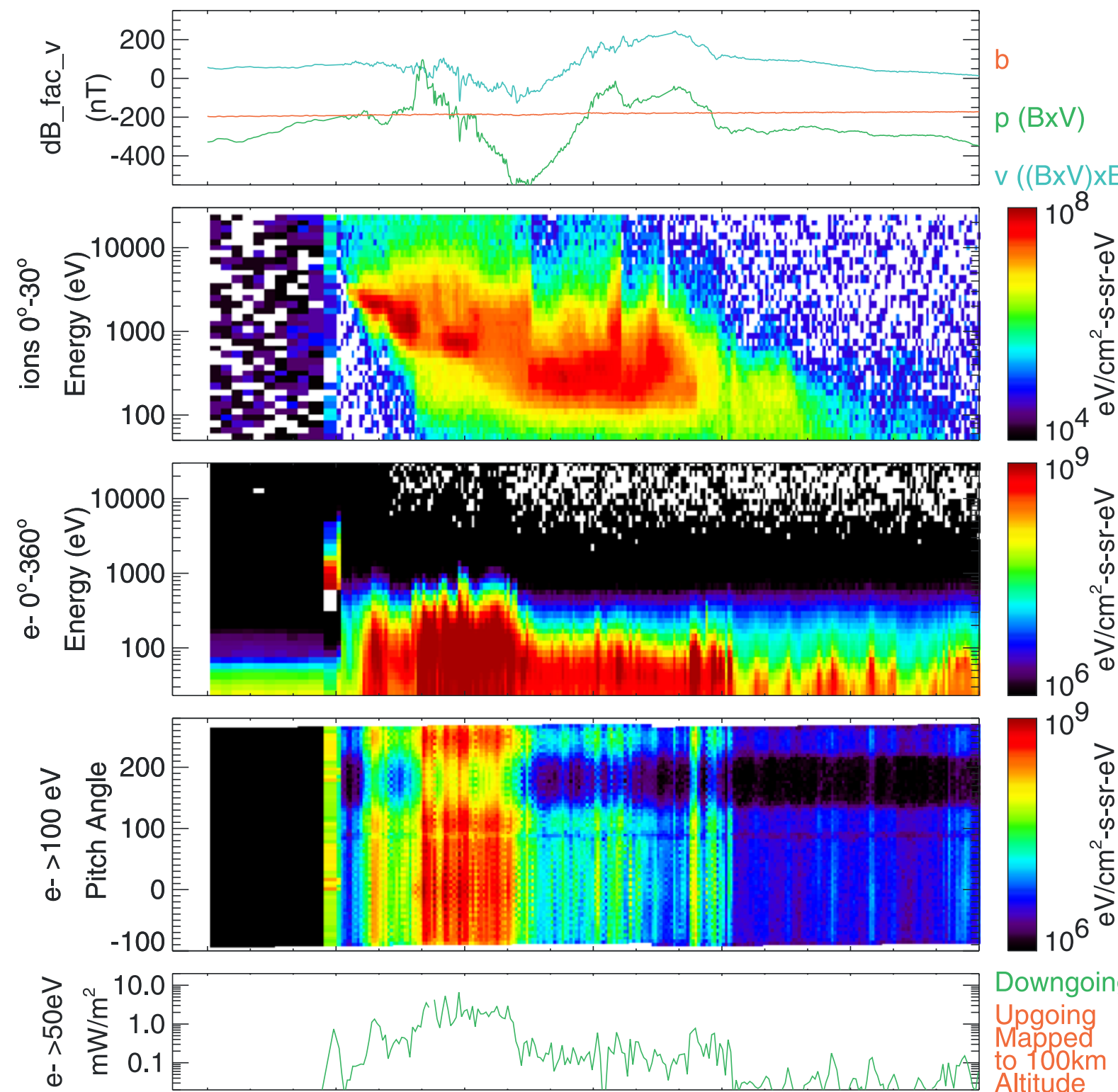

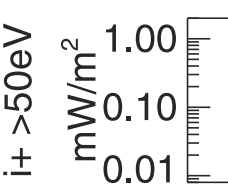

Upgoing : Mapped

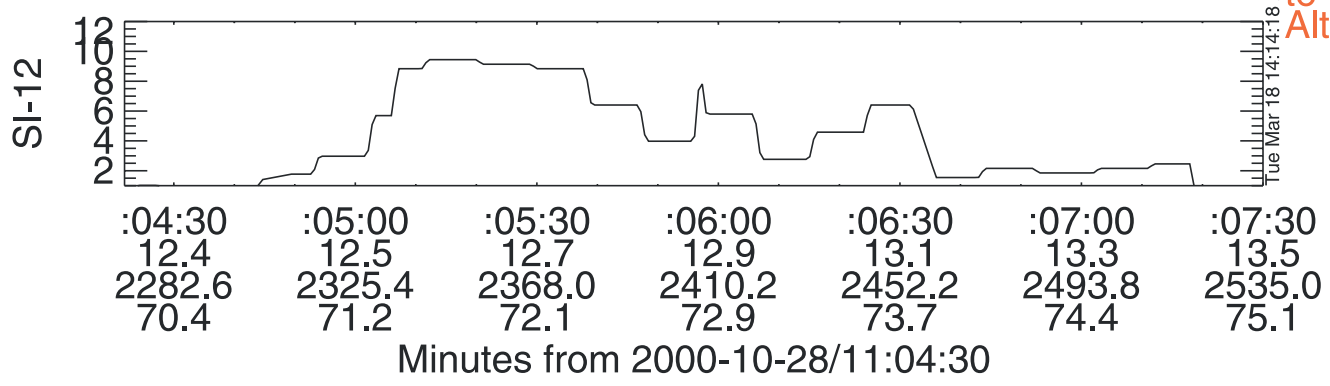




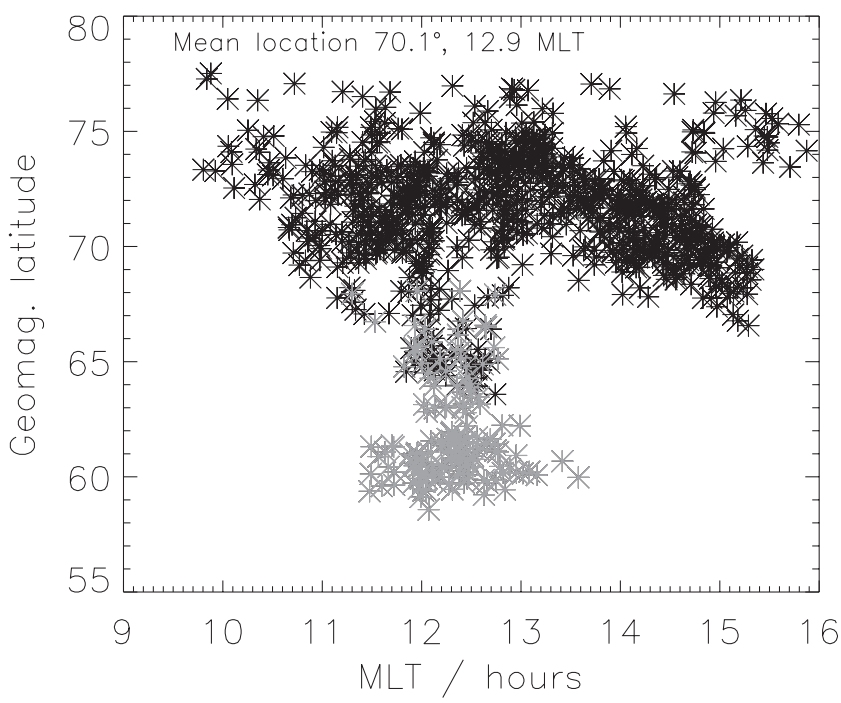

Figure 8. Distribution of cusp aurora observations in magnetic local time and geomagnetic latitude. The mean values are $70.1^{\circ}$ latitude and 12.9 hours magnetic local time.

decreased. Caution has to be used in the physical interpretation of this result. The SI-12 instrument can not determine the exact amount of Doppler shift (and energy of the precipitating proton). Precipitating protons in the cusp have energies around $2 \mathrm{keV}$. The SI-12 instrument is mostly sensitive to protons of $2-8 \mathrm{keV}$ energy and has a steep sensitivity decrease below $2 \mathrm{keV}$ [see Gérard et al., 2001, Figure 2]. The temporal decrease of the cusp proton brightness in SI-12 can thus be caused by either a constant energy and proportional decrease in precipitating flux or by just a small decrease in energy with constant precipitating flux. In our view a small energy decrease is the more likely cause of the observed signal decrease during stable external IMF conditions.

[33] There are two major differences between the present results and those of the earlier study of northward IMF proton aurora [Frey et al., 2002]. First, in the present study the geomagnetic location of the bright proton aurora strongly depends on the IMF $\mathrm{B}_{z}$ magnitude with an average displacement of 0.45 degrees/nT and a correlation coefficient of 0.81 . In the northward IMF study there was no significant dependence between the two quantities $(\mathrm{R}=0.08)$. This result is in agreement with the results of Newell et al. [1989], who also found a good correlation for southward IMF $(\mathrm{R}=0.70)$ and no correlation for the northward case $(\mathrm{R}=0.18)$. This result can be understood in terms of the magnetospheric field line topology (Figure 12) if we assume that during antiparallel reconnection the magnitude of the solar wind magnetic field plays a role in the determination of the reconnection site. During southward IMF conditions, reconnection in the lowlatitude region erodes closed magnetic field lines [Burch, 1973] and moves the low-latitude magnetopause and recon-
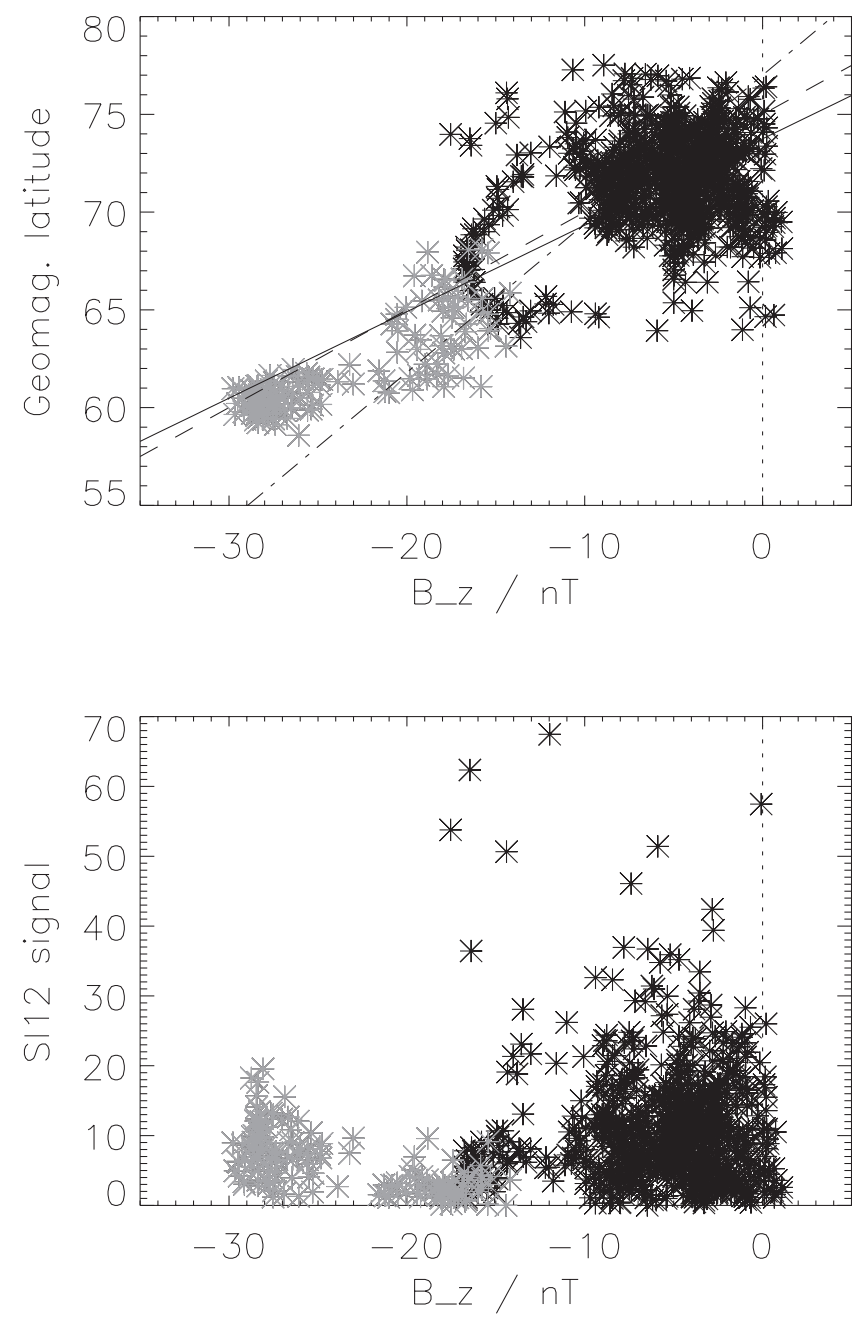

Figure 9. Dependence of the latitude location and proton aurora brightness on the value of the IMF $\mathrm{B}_{z}$. The solid line is the least square fit of $73.8^{\circ}+0.45^{\circ} B_{z}$. The dashed-dotted line is the result from Newell et al. [1989]. The dashed line is the result from Sandholt et al. [1994].

nection site closer to the Earth to a region with stronger magnetic field. The general shape of these field lines is very similar to those reconnected earlier, and a motion inward will only minimally change the angle between the magnetosheath and the magnetosphere field lines, and therefore antiparallel reconnection is still expected to occur at that location. A strong external field reconnects efficiently to those field lines with larger magnetic field strength and will therefore be connected to lower latitudes. During northward IMF conditions, reconnection occurs with high-latitude closed field lines [Onsager and Fuselier, 1994]. Reconnection with the external field will erode those field lines which fulfill the antiparallel condition. The further inward located field lines have different shapes and direction. Therefore the antiparallel

Figure 7. (opposite) Summary of FAST measurements and FUV observations on 28 October 2000. The top panel shows the magnetic field disturbances with the green line representing the cross-track component. The next panel is the ion energy spectrogram in the loss cone. Then follow the electron energy spectrogram averaged over all pitch angles, and the pitch angle distribution. The next two panels summarize the energy fluxes of electrons and ions, mapped to $100 \mathrm{~km}$ altitude. The last panel shows the count rate in the three consecutive SI-12 images along the footprint of FAST. 

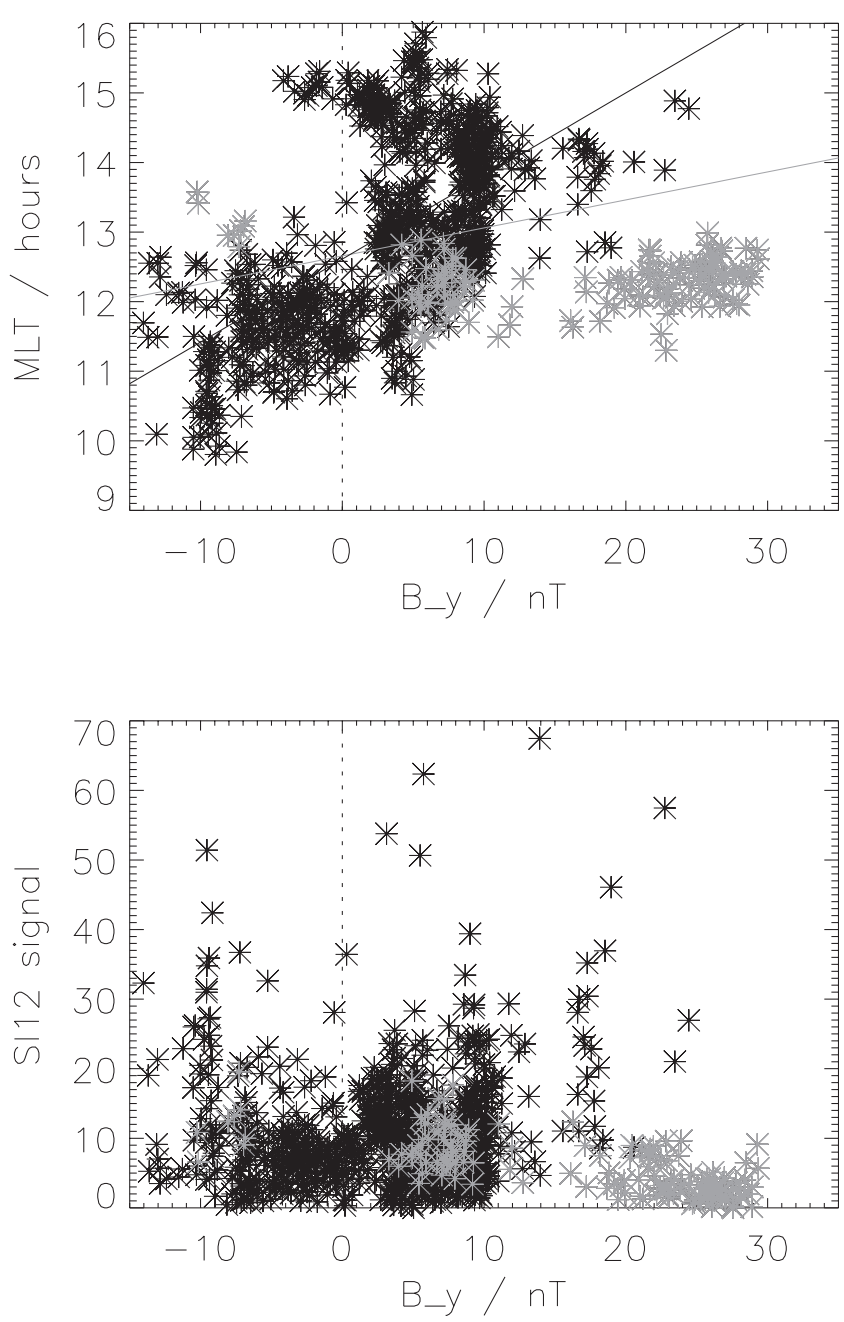

Figure 10. Dependence of the local time location and proton aurora brightness on the value of IMF $\mathrm{B}_{y}$. The top panel also shows the least square fitted linear relation for the full data set (gray line) and without data from 12 August (black line).

condition is not fulfilled and the reconnection site can not move to field lines with more poleward foot prints.

[34] The other difference between the northward and southward cases is the difference in brightness of the proton aurora for the same solar wind dynamic pressure. Equation (3) shows a linear coefficient of 1.51 while the linear coefficient in the northward case was 1.2. Gas dynamic models of the magnetosheath predict variations of the plasma parameters as a function of position in the magnetosheath [Walker and Russell, 1995], with 4X the solar wind density, 20X the solar wind temperature and zero velocity at the nose of the magnetopause, and values very similar to the solar wind properties at high latitudes far down the tail. The observed increase of the proton aurora brightness from lowlatitude reconnection by about $25 \%$ can not be explained by the increased density and temperatures in the low-latitude region, especially not as the source plasma should have zero velocity. One additional factor is the existence of a plasma depletion layer at the magnetosheath-magnetopause interface [Zwan and Wolf, 1976]. The squeezing of the magnetic field reduces the magnetosheath plasma density even in the absence of reconnection at low [Anderson et al., 1997] and at high latitudes [Fuselier et al., 2000]. It is rather the reconnection process itself which accelerates the protons to energies sufficient for the excitation of proton aurora [Smith et al., 1992]. There is one more effect in the high-latitude region which will play a role in our investigation. The solar wind flow past the magnetosphere creates a general antisunward flow in the magnetosheath. Reconnection in the lowlatitude region creates reconnected field lines moving in the same direction as the magnetosheath flow, while highlatitude reconnection creates reconnected field lines which move against the general flow. This difference can well explain the observed difference of about $25 \%$ larger proton aurora brightness if the background flow adds to the convection of reconnected flux tubes compared to the case when the reconnected flow has to act against the background flow.

[35] There is some indication of a weak dependence of the latitude location of the proton aurora on the solar wind dynamic pressure (Figure 11) [Mende et al., 1998]. However, for the whole data set, the correlation is very poor with a coefficient of only 0.23 .

[36] The observation of a four times increase in Dopplershifted Lyman- $\alpha$ brightness in the single case study by
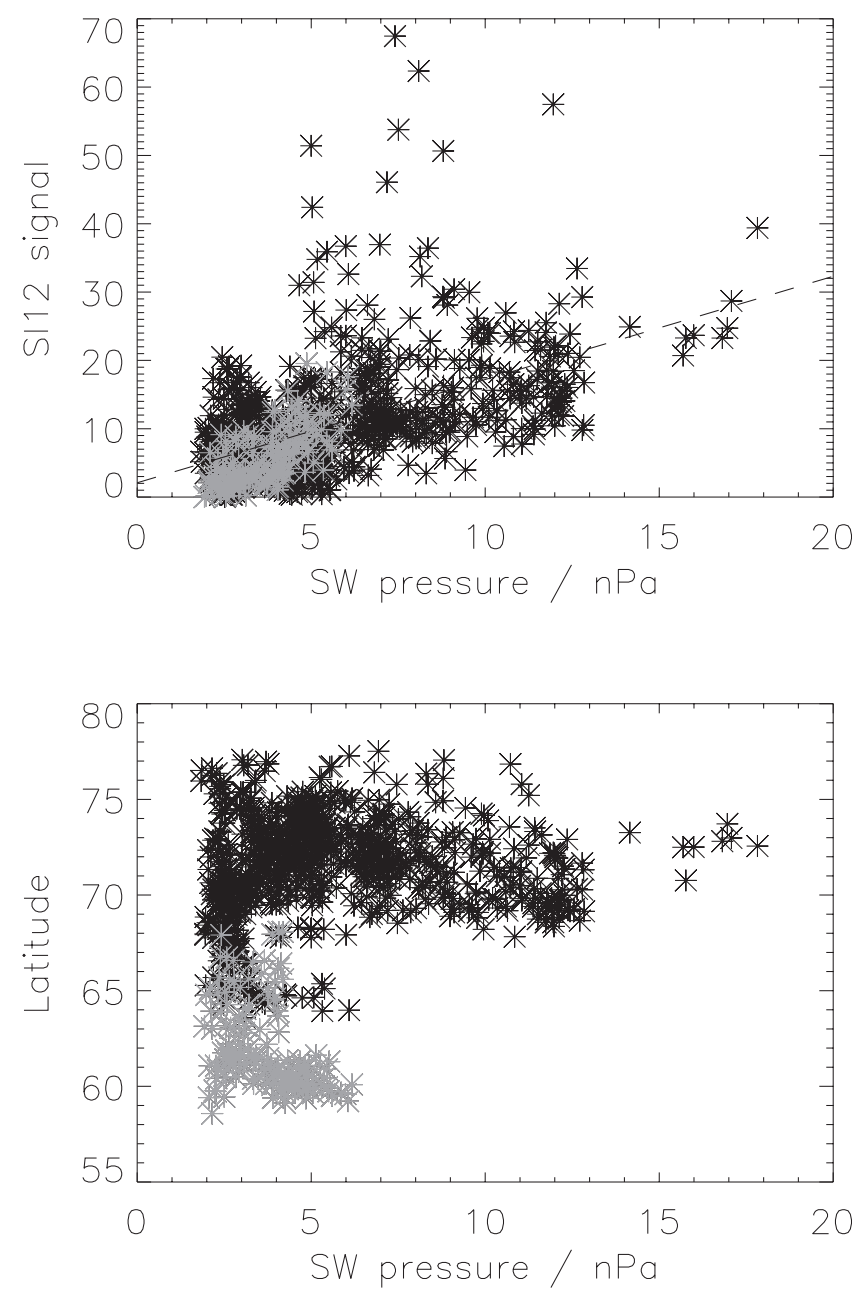

Figure 11. Dependence of the latitude location and proton aurora brightness on the solar wind dynamic pressure. The top panel also shows the least square fitted linear relation of $\mathrm{SI} 12=2.0+1.51 \mathrm{p}_{d y n}$. 

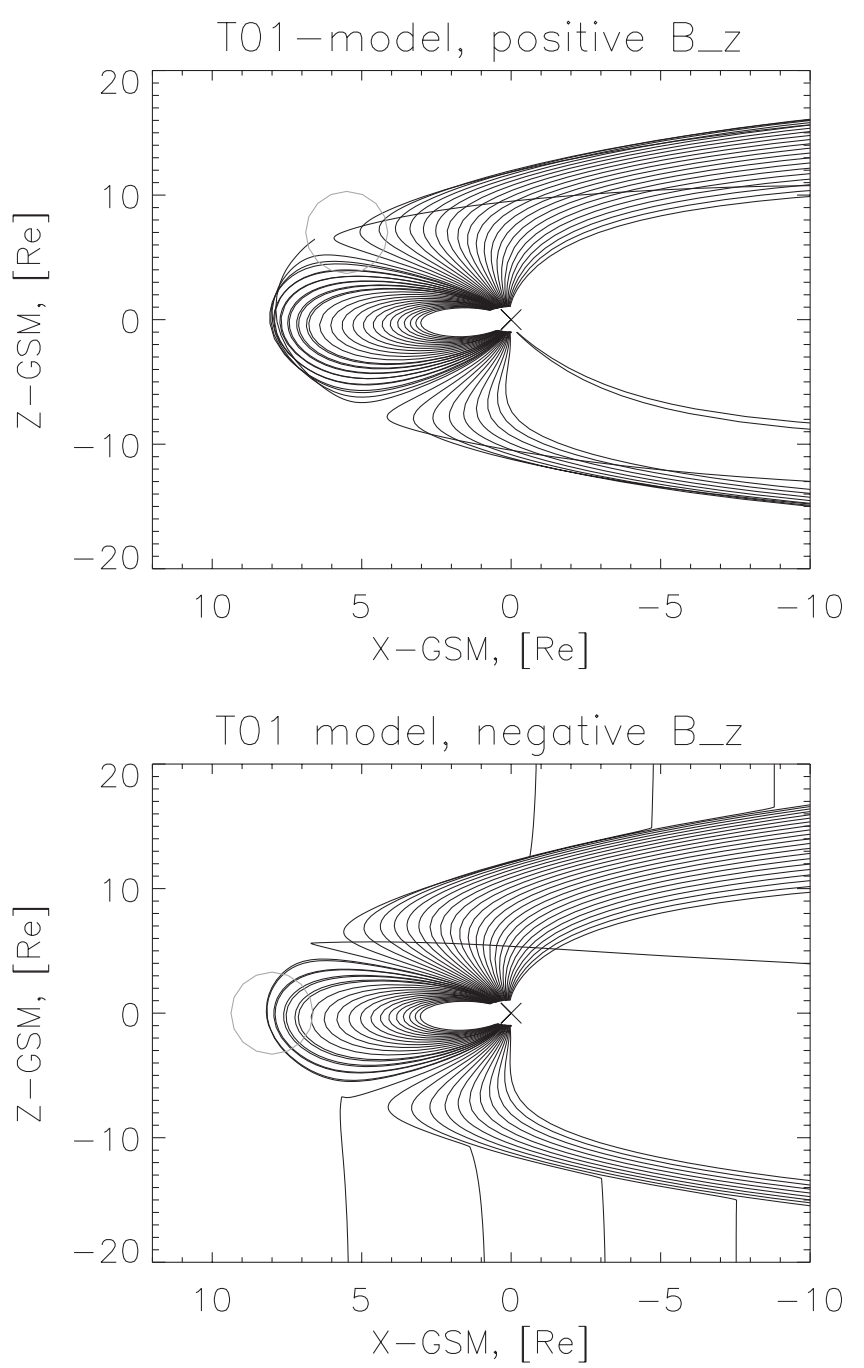

Figure 12. Magnetospheric field lines during northward IMF ( $5 \mathrm{nT}$, top) and southward IMF ( $-5 \mathrm{nT}$, bottom) for typical conditions of this study with Dst $=-20 \mathrm{nT}, \mathrm{P}_{d y n}=7$ $\mathrm{nPa}, \mathrm{B}_{x}=-2 \mathrm{nT}, \mathrm{B}_{y}=0 \mathrm{nT}$ [Tsyganenko, 2002]. Circles indicate the location of antiparallel regions for reconnection during the northward and southward IMF conditions.

Lockwood et al. [2003] seems to contradict our result of just a $25 \%$ increase of Lyman- $\alpha$ brightness from northward to southward IMF conditions. However, there are three major differences in both approaches. In our case we determine this increase from the statistical analysis of many cases and not just one. The statistical average is very likely lower than the result of one extreme case. Second, we compare the average brightness in an area of $500 \times 500 \mathrm{~km}^{2}$ around the brightest pixel in the SI-12 images, while Lockwood et al. [2003] investigate the brightest pixel only. Third, we compare the behavior of the Lyman- $\alpha$ emission over long time periods of at least $20 \mathrm{~min}$, while the short-lived southward turning in the Lockwood et al. investigation lasted for only $5 \mathrm{~min}$. We have discussed several examples when the Lyman- $\alpha$ brightness immediately after the IMF southward turn increased dramatically but over time decreased, even for slowly increasing solar wind dynamic pressure. Therefore we consider both results in general agreement.

\section{Conclusions}

[37] We performed a statistical investigation of the properties of the proton aurora at the cusp footprint during southward IMF conditions as frequently observed by the FUV instrument on IMAGE. This investigation extends an earlier study of a strong spot-like proton aurora signature of cusp precipitation during northward IMF conditions and confirms a few earlier established relationships between the cusp proton aurora and IMF conditions and also established a few new results.

[38] 1. The previously described correlation between the magnetic local time location and the IMF $\mathrm{B}_{y}$ magnitude [see, e.g., Newell et al., 1989; Sandholt and Farrugia, 1999; Frey et al., 2002] were confirmed. If one particular day of extreme IMF $B_{z}$ values were excluded form the present data set, a linear relationship of 0.120 hours/ $B_{y}$ was found that is very consistent with the value of 0.127 hours $/ B_{y}$ for the northward IMF case [Frey et al., 2002].

[39] 2. The previously described relationship between the solar wind dynamic pressure and the brightness of the Doppler-shifted Lyman- $\alpha$ emission was again established. As in the northward IMF case [Frey et al., 2002], only a small correlation coefficient of 0.55 could be found because the Lyman- $\alpha$ signal tends to decrease with time for constant or even slightly increasing external pressure conditions. This suggests that the proton aurora brightness does not just depend on the instantaneous solar wind pressure value but also on the past history of it. A better correlation coefficient of 0.68 is found if only those values immediately following pressure increases were used.

[40] 3. There is an average $9^{\circ}$ lower latitude location of the cusp footprint than in the northward IMF case [Frey et $a l ., 2002]$ which also verifies previous results from groundbased and satellite studies [see, e.g., McWilliams et al., 2001; Newell et al., 1989]. In contrast to the northward IMF case [Frey et al., 2002], a strong correlation exists between the magnitude of $\mathrm{B}_{z}$ and the latitude position of the bright proton aurora at the cusp footprint (correlation coefficient 0.81 ). It is unclear why this study found different linear relationships than earlier studies of in situ particle signatures [Carbary and Meng, 1986; Newell et al., 1989]. The very good agreement with results from a ground-based optical investigation [Sandholt et al., 1994] suggests that different selection criteria for particle and optical observations may be the reason for this difference.

[41] 4. The new result of this study is the larger proton aurora brightness for southward IMF compared with the northward case. For similar external pressures a $25 \%$ increase in Doppler-shifted Lyman- $\alpha$ brightness was determined for southward IMF cases. The most likely cause of this increase is a combination of instrument properties and physical effect. Precipitating protons in the cusp have mean energies around $2 \mathrm{keV}$, and they tend to fall into the energy range of the instrument response, where small changes in energy may cause rather large changes in instrument counts. Low-latitude reconnection during southward IMF creates flux tubes that convect in the same sense as the magnetosheath flow. This is in contrast to high-latitude lobe reconnection during northward IMF which creates flux tubes convecting against the magnetosheath flow [Onsager and Lockwood, 1997]. The additional energy gain for flux tubes 
moving in the same direction as the background magnetosheath flow can then well explain the slightly larger proton aurora brightness during southward IMF.

[42] Together with the earlier studies [Frey et al., 2002; Fuselier et al., 2002], this study summarizes major properties of the cusp proton aurora occurrence, brightness, and location. Several more open questions require further investigation, as for instance dipole and seasonal effects on the brightness of the proton aurora. The combination of in situ satellite observations and proton aurora images will be important in establishing the exact energy of precipitating protons and how this affects the brightness of Doppler-shifted Lyman- $\alpha$ emission. The progress of the IMAGE apogee toward the equatorial plane will offer more opportunities in the future for such investigations when the Northern and the Southern Hemispheres may be studied from closer distances.

[43] Acknowledgments. We are grateful to the IMAGE-SMOC team who keep the IMAGE spacecraft running. The IMAGE FUV investigation was supported by NASA through SwRI subcontract 83820 at the University of California at Berkeley under contract NAS5-96020. The solar wind measurements were obtained from CDAWeb. We acknowledge the following PIs: WIND Magnetic Fields Investigation, R. Lepping; WIND Solar Wind Experiment, K. Ogilvie; Geotail Magnetic Field Instrument, S. Kokubun; Geotail Comprehensive Plasma Instrument, L. Frank; ACE Magnetic Field Data, N. Ness, Bartol Research Institute; ACE Solar Wind Experiment, D. J. McComas, Southwest Research Institute.

[44] Arthur Richmond thanks Per Even Sandholt and another reviewer for their assistance in evaluating this paper.

\section{References}

Anderson, B. J., T.-D. Phan, and S. A. Fuselier, Relationships between plasma depletion and subsolar reconnection, J. Geophys. Res., 102, 9531, 1997

Burch, J. L., Rate of erosion of dayside magnetic flux based on a quantitative study of polar cusp latitude on the interplanetary magnetic field, Radio Sci., 8, 955, 1973.

Carbary, J. F., and C. I. Meng, Correlation of cusp latitude with $\mathrm{B}_{z}$ and AE(12) using nearly one year's data, J. Geophys. Res., 91, 10,047, 1986.

Coleman, I. J., M. Pinnock, and A. S. Rodger, The ionospheric footprint of anti-parallel merging regions on the dayside magnetopause, Ann. Geophys., 18, 511, 2000.

Cowley, S. W. H., Magnetospheric asymmetries associated with the Y-component of the IMF, Planet. Space Sci., 29, 79, 1981.

Frey, H. U., S. B. Mende, C. W. Carlson, J.-C. Gérard, B. Hubert, J. Spann, R. Gladstone, and T. J Immel, The electron and proton aurora as seen by IMAGE-FUV and FAST, Geophys. Res. Lett., 28, 1135, 2001.

Frey, H. U., S. B. Mende, T. J. Immel, S. A. Fuselier, E. S. Claflin, J.-C. Gérard, and B. Hubert, Proton aurora in the cusp, J. Geophys. Res., 107(A7), 1091, doi:10.1029/2001JA900161, 2002.

Frey, H. U., S. B. Mende, T. J. Immel, J.-C Geŕard, B. Hubert, S. Habraken, J. Spann, G. R. Gladstone, D. V. Bisikalo, and V. I. Shematovich, Summary of quantitative interpretation of IMAGE far ultraviolet data, Space Sci. Rev., in press, 2003.

Fuselier, S. A., S. M. Petrinec, and K. J. Trattner, Stability of the highlatitude reconnection site for steady northward IMF, Geophys. Res. Lett., 27, 473, 2000.

Fuselier, S. A., H. U. Frey, K. J. Trattner, S. B. Mende, and J. L. Burch, Cusp aurora dependence on IMF $\mathrm{B}_{z}$, J. Geophys. Res., 107(A7), 1111, doi:10.1029/2001JA900165, 2002.

Gérard, J.-C., B. Hubert, D. V. Bisikalo, and V. I. Shematovich, A model of the Lyman- $\alpha$ line profile in the proton aurora, J. Geophys. Res., 105, $15,795,2000$.

Gérard, J.-C., B. Hubert, M. Meurant, V. I. Shematovich, D. V. Bisikalo, H. Frey, S. Mende, G. R. Gladstone, and C. W. Carlson, Observation of the proton aurora with IMAGE FUV imager and simultaneous ion flux in situ measurements, J. Geophys. Res., 106, 28,939, 2001.

Hubert, B., J.-C. Gérard, D. S. Evans, M. Meurant, S. B. Mende, H. U. Frey, and T. J. Immel, Total electron and proton energy input during auroral substorms: Remote sensing with IMAGE-FUV, J. Geophys. Res., 107(A8), 1183, doi:10.1029/2001JA009229, 2002.

Jacobsen, B., P. E. Sandholt, W. J. Burke, W. F. Denig, and N. C. Maynard, Optical signatures of prenoon auroral precipitation: Sources and responses to solar wind variations, J. Geophys. Res., 100, 8003, 1995.
Lockwood, M., B. S. Lanchester, H. U. Frey, K. Throp, S. K. Morley, S. E. Milan, and M. Lester, IMF control of cusp proton emission intensity and dayside convection: Implications for component and anti-parallel reconnection, Ann. Geophys., 21(4), 955, 2003.

McCrea, I. W., M. Lockwood, J. Moen, F. Pitout, P. Eglitis, A. D. Aylward, J.-C. Cerisier, A. Thorolfssen, and S. E. Milan, ESR and EISCAT observations of the response of the cusp and cleft to IMF orientation changes, Ann. Geophys., 18, 1009, 2000.

McWilliams, K. A., T. K. Yeoman, J. B. Sigwarth, L. A. Frank, and M. Brittnacher, The dayside ultraviolet aurora and convection responses to a southward turning of the interplanetary magnetic field, Ann. Geophys., $19,707,2001$

Mende, S. B., D. M. Klumpar, S. A. Fuselier, and B. J. Anderson, Dayside auroral dynamics: South Pole-AMPTE/CCE observations, J. Geophys. Res., 103, 6891, 1998.

Mende, S. B., et al., Far Ultraviolet imaging from the IMAGE spacecraft, Space Sci. Rev., 91, 287, 2000.

Moen, J., H. C. Carlson, and P. E. Sandholt, Continuous observation of cusp auroral dynamics in response to an IMF $\mathrm{B}_{y}$ polarity change, Geophys. Res. Lett., 26, 1243, 1999.

Newell, P. T., C.-I. Meng, D. G. Sibeck, and R. Lepping, Some low-altitude cusp dependencies on the interplanetary magnetic field, J. Geophys. Res., 94, 8921, 1989.

Newell, P. T., W. J. Burke, C.-I. Meng, E. R. Sanchez, and M. E. Greenspan, Identification and observations of the plasma mantle at low altitude, J. Geophys. Res., 96, 35, 1991.

Onsager, T. G., and S. A. Fuselier, The location of magnetic reconnection for southward and northward interplanetary magnetic field, in Solar System Plasmas in Space and Time, Geophys. Monogr. Ser., vol. 84, edited by J. L. Burch and J. H. Waite Jr., p. 183, AGU, Washington, D.C., 1994. Onsager, T. G., and M. Lockwood, High-latitude particle precipitation and its relationship to magnetospheric source regions, Space Sci. Rev., 80, 77, 1997.

Onsager, T. G., C. A. Kletzing, J. B. Austin, and H. MacKiernan, Model of magnetosheath plasma in the magnetosphere: Cusp and mantle particles at low altitudes, Geophys. Res. Lett., 20, 479, 1993.

Onsager, T. G., S.-W. Chang, J. D. Perez, J. B. Austin, and L. X. Janoo, Low-altitude observations and modeling of quasi-steady magnetopause reconnection, J. Geophys. Res., 100, 11,831, 1995.

Sandholt, P. E., and C. J. Farrugia, On the dynamic cusp aurora and IMF $\mathrm{B}_{y}$, J. Geophys. Res., 104, 12,461, 1999.

Sandholt, P. E., et al., Cusp/cleft auroral activity in relation to solar wind dynamic pressure, interplanetary magnetic field $\mathrm{B}_{z}$ and $\mathrm{B}_{y}, J$. Geophys. Res., 99, 17,323, 1994.

Sandholt, P. E., C. J. Farrugia, J. Moen, O. Noraberg, B. Lybekk, T. Sten, and T. Hansen, A classification of dayside auroral forms and activities as a function of interplanetary magnetic field orientation, J. Geophys. Res., 103, 23,325, 1998.

Sandholt, P. E., C. J. Farrugia, S. W. H. Cowley, M. Lester, and J.-C. Cerisier, Excitation of transient lobe cell convection and auroral arc at the cusp poleward boundary during a transition of the interplanetary magnetic field from south to north, Ann. Geophys., 19, 487, 2001.

Smith, M. F., and M. Lockwood, Earth's magnetospheric cusps, Rev. Geophys., 34, 233, 1996.

Smith, M. F., M. Lockwood, and S. W. H. Cowley, The statistical cusp: A flux transfer model, Planet. Space Sci., 40, 1251, 1992.

Tsyganenko, N. A., A model of the near magnetosphere with a dawn-dusk asymmetry: 2. Parameterization and fitting to observations, J. Geophys. Res., 107(A8), 1176, doi:10.1029/2001JA000220, 2002.

Walker, R. J., and C. T. Russell, Solar-wind interactions with magnetized planets, in Introduction to Space Physics, edited by M. G. Kivelson and C. T. Russell, p. 178, Cambridge Univ. Press, New York, 1995.

Woch, J., and R. Lundin, Magnetosheath plasma precipitation in the polar cusp and its control by the interplanetary magnetic field, J. Geophys. Res., 97, 1421, 1992.

Yeoman, T. K., P. G. Hanlon, and K. A. McWilliams, A statistical study of the location and motion of the HF radar cusp, Ann. Geophys., 20, 275, 2002.

Zwan, B. J., and R. A. Wolf, Depletion of solar wind plasma near a planetary boundary, J. Geophys. Res., 81, 1636, 1976.

H. U. Frey, T. J. Immel, S. B. Mende, and N. Østgaard, Space Sciences Laboratory, University of California, Berkeley, CA 94720-7450, USA. (hfrey@ssl.berkeley.edu; immel@ssl.berkeley.edu; mende@ssl.berkeley. edu; nikost@ssl.berkeley.edu)

S. A. Fuselier, Lockheed Martin ATC, 3251 Hanover St., Palo Alto, CA 94304-1191, USA. (stephen.a.fuselier@1mco.com) 